\title{
metaFALCON: A program package for automatic sampling of conical intersection seams using multistate metadynamics
}

\author{
Joachim O. Lindner, ${ }^{\dagger}$ Karina Sultangaleeva, ${ }^{\dagger}$ Merle I. S. Röhr, ${ }^{*, \dagger}, \dagger$ and Roland \\ Mitrić*, \\ $\dagger$ Institut für Physikalische und Theoretische Chemie, Julius-Maximilians-Universität \\ Würzburg, Emil-Fischer-Str. 42, 97074 Würzburg, Germany \\ $\ddagger$ Center for Nanosystems Chemistry (CNC), Julius-Maximilians-Universität Würzburg, \\ Theodor-Boveri-Weg, 97074 Würzburg, Germany
}

E-mail: merle.roehr@uni-wuerzburg.de; roland.mitric@uni-wuerzburg.de

\begin{abstract}
The multistate metadynamics for automatic exploration of conical intersection seams and systematic location of minimum energy crossing points in molecular systems and its implementation into the software package metaFALCON is presented. Based on a locally modified energy gap between two Born-Oppenheimer electronic states as a collective variable, multistate metadynamics trajectories are driven towards an intersection point starting from an arbitrary ground state geometry and are subsequently forced to explore the conical intersection seam landscape. For this purpose, an additional collective variable capable of distinguishing structures within the seam needs to be defined and an additional bias is introduced into the off-diagonal elements of an extended (multistate) electronic Hamiltonian. We demonstrate the performance of the
\end{abstract}


algorithm on the examples of the 1,3-butadiene, benzene and 9H-adenine molecules, where multiple minimum energy crossing points could be systematically located using the Wiener number or Cremer-Pople parameters as collective variables. Finally, on the example of $9 \mathrm{H}$-adenine we show that the multistate metadynamics potential can be used to obtain a global picture of a conical intersection seam. Our method can be straightforwardly connected with any ab initio or semiempirical electronic structure theory that provides energies and gradients of the respective electronic states and can serve for systematic elucidation of the role of conical intersections in the photophysics and photochemistry of complex molecular systems, thus complementing nonadiabatic dynamics simulations.

\section{Introduction}

The concept of adiabatic potential energy surfaces (PES), the source of the fundamental concepts of chemistry, is rooted in the Born Oppenheimer (BO) approximation. For the majority of chemical reactions taking place in the ground electronic state, the latter allows for the theoretical treatment of reactivity, the identification of reaction mechanisms as well as the prediction of kinetic parameters.

However, in photochemical processes involving electronically excited states the character of the electronic wavefunction may rapidly change with the nuclear configuration as the reaction proceeds, leading to the complete breakdown of the BO approximation. The change of the character of the electronic wavefunction leads to a large nonadiabatic coupling being responsible for efficient non-radiative transitions between electronic states. These couplings are largest in the vicinity of conical interesections (CIs) between the adiabatic potential energy surfaces. ${ }^{1-4}$ The role of CIs in photochemistry, photophysics and photobiology is reflected in countless examples, such as vision, ${ }^{5,6}$ the photostability of DNA, ${ }^{7}$ organic photochemical synthesis ${ }^{8}$ as well as the functionality of photovoltaic devices, ${ }^{9}$ and a significant effort has been undertaken to identify them experimentally using various spectroscopic techniques. ${ }^{6,10,11}$ 
Therefore, in order to predict the fait of an electronically excited molecule it is crucial to theoretically characterize structure, energetics and reaction pathways leading to conical intersections. Several strategies have been applied for the optimization of minimum energy crossing points (MECP) based on penalty functions, ${ }^{12}$ gradient projection ${ }^{13}$ or LagrangeNewton techniques. ${ }^{14}$ All these methods require the knowledge of a reasonable initial guess that can be generated either by chemical intuition or, for example, by running nonadiabatic dynamics simulations. Although even an inaccurate guess may lead to some MECP, it is not clear which role this structure plays in the context of the complete crossing seam. Attempts to characterize the full intersection seam instead of individual geometries have so far relied on symmetry considerations ${ }^{15}$ or the use of algorithms such as the nudged elastic band (NEB) method ${ }^{16}$ or anharmonic downward distortion following (ADDF). ${ }^{17-20}$ Furthermore, transition states on the intersection seam have been adressed by an analytical second-order description of the intersection seam and used for linking multiple crossing points. ${ }^{21,22}$

In order to drive a molecule from a ground state minimum to the lowest intersection points, the energy has to be raised by several $\mathrm{eV}$, which is in the scope of what can be achieved by accelerated molecular dynamics (MD) techniques. The metadynamics introduced by Parrinello and coworkers ${ }^{23-28}$ represents an enhanced sampling approach that is both easy to use and efficient. This recently lead us to introduce a multistate extension of the metadynamics that is suitable for a fully automatic exploration of conical intersection seams. ${ }^{29}$ Usually, the main challenge in applying the metadynamics method is to find suitable collective variables (CV) capable of driving transitions between different basins on the PES. Consequently, much effort has been put in the definition of suitable CVs, that can in principle be as simple as a single bond length but sometimes even require advanced techniques such as machine learning or dimensionality reduction to be generated. ${ }^{27,30}$

In our multistate metadynamics, we use a locally modified energy gap as CV, which is applicable to any molecular system, augmented by an additional geometric CV which drives seam exploration. In this article, we first provide a detailed formulation of the mul- 
tistate metadynamics and its implementation within our newly developed metaFALCON

package. In order to illustrate the method and test its robustness with respect to the choice of the simulation parameters we apply it to the well studied 1,3-butadiene and benzene molecules whose intersection seams have been thoroughly characterized before. ${ }^{15,31-37}$ Finally, we demonstrate on the example of the DNA base $9 \mathrm{H}$-adenine that a full intersection seam can be reconstructed based on the multistate metadynamics.

\section{Methods}

Multistate metadynamics algorithm. The idea of the multistate metadynamics ${ }^{29}$ is to use the energy gap between the ground and excited electronic states as a collective variable serving to drive the molecule toward the CI seam starting from an arbitrary ground state structure (e.g. the ground state minimum). The system is propagated using the Newtonian equations of motion augmented by an additional history-dependent bias potential $V_{G}(t)$, which for the $i$-th particle read:

$$
m_{i} \ddot{\mathbf{R}}_{i}=-\nabla_{i}\left(E_{g}+V_{G}(t)\right)
$$

Here, $E_{g}$ represents the ground state BO PES. The history-dependent bias potential is updated at regular time steps $\tau_{G}$ by adding Gaussian-shaped functions along the energy gap CV defined further below:

$$
\begin{aligned}
V_{G}(t)= & \sum_{t^{\prime}=\tau_{G}, 2 \tau_{G}, \ldots}^{t} w \exp \left(-\frac{\left(\Delta E_{\text {meta }}(t)-\Delta E_{\text {meta }}\left(t^{\prime}\right)\right)^{2}}{2 \delta s^{2}}\right) \\
& \times \Theta\left(\Delta E_{\text {meta }}\left(t^{\prime}\right)-\epsilon\right),
\end{aligned}
$$

The bias potential is dependent on the modified energy gap $\Delta E_{\text {meta }}$, and $w$ and $\delta s$ represent the fixed height and width, respectively. An important feature of the algorithm is that the bias potential is updated only if the value of the gap is larger than a numerical threshold $\epsilon$. 

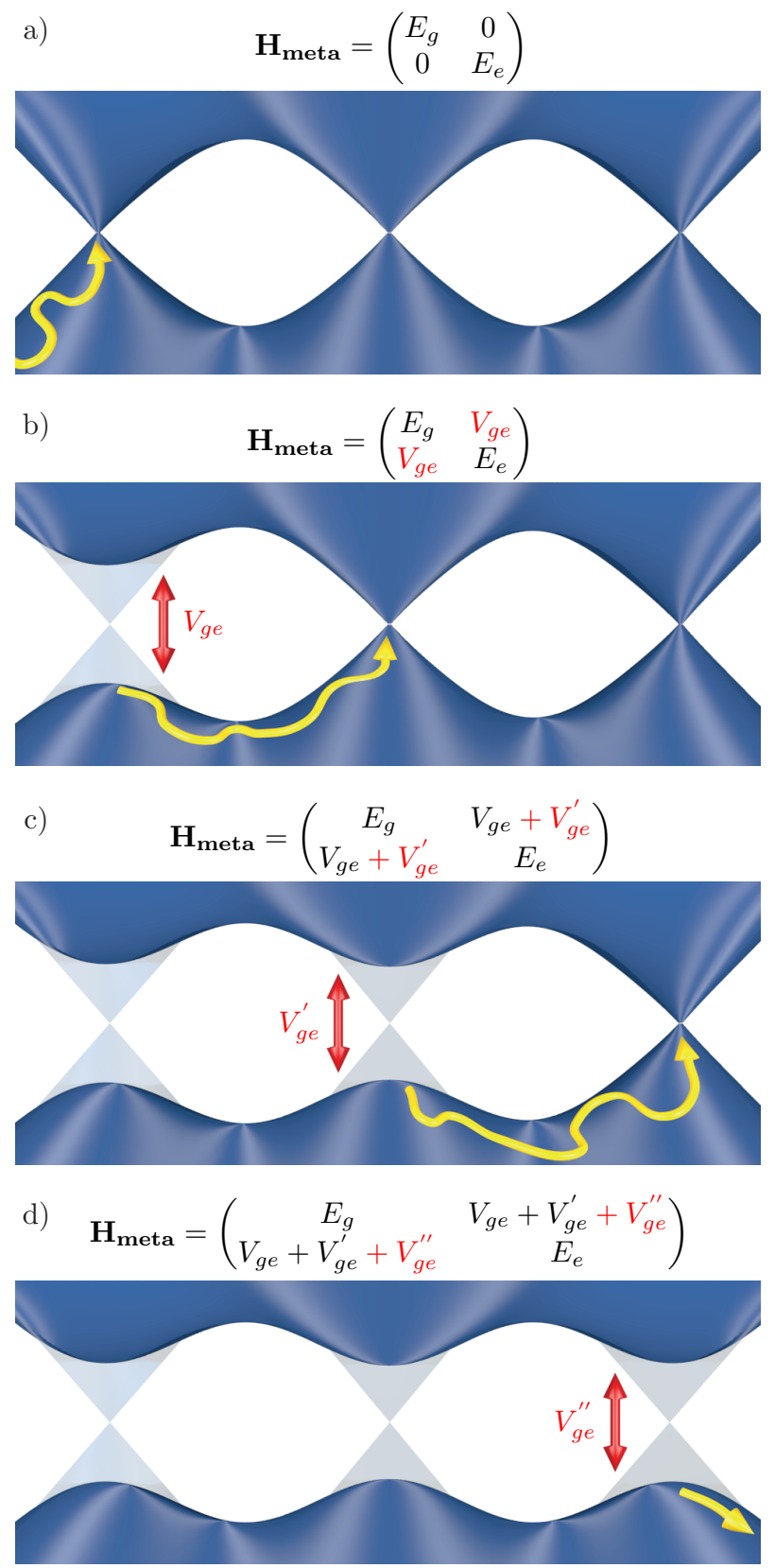

Figure 1: Scheme of the algorithm. (a) The bias potential $V_{G}$ is constructed in order to drive the system from lower-energy regions on the ground state PES toward the intersection seam (yellow trajectory). (b) Upon reaching the seam, an off-diagonal coupling $V_{g e}$ (red) is added to the electronic Hamiltonian, opening the effective energy gap. The metadynamics subsequently reduces the impact of $V_{g e}$ which leads to the next intersection point. The addition of the further bias potentials $V_{g e}^{\prime}(\mathrm{c})$ and $V_{g e}^{\prime \prime}(\mathrm{d})$ enables the complete exploration of the CI seam. 
This is enforced by the presence of the Heaviside theta function $\Theta\left(\Delta E_{\text {meta }}-\epsilon\right)$ in Eq. 2 .

Starting a molecular dynamics simulation e.g. from a minimum on the ground state PES, the bias potential will force the system to move toward the CI seam by systematically lowering the value of the energy gap (see Fig. 1a for illustration). Upon reaching the intersection seam for the first time, the molecular electronic Hamiltonian, which is initially diagonal in the BO approximation, is extended by introducing a further biasing potential $V_{g e}$ into the off-diagonal elements according to

$$
\mathbf{H}_{\mathbf{B O}}=\left(\begin{array}{cc}
E_{g} & 0 \\
0 & E_{e}
\end{array}\right) \rightarrow \mathbf{H}_{\text {meta }}=\left(\begin{array}{cc}
E_{g} & V_{g e} \\
V_{g e} & E_{e}
\end{array}\right)
$$

This is a crucial element of our algorithm, leading to a local modification of the PES, which is obtained by diagonalization of $\mathbf{H}_{\text {meta }}$. The metadynamics continues to run on a such locally modified potential energy surface with the effective energy gap

$$
\Delta E_{m e t a}=\sqrt{\left(E_{g}-E_{e}\right)^{2}+4 V_{g e}^{2}}
$$

The gap between the eigenstates of the modified Hamiltonian is enhanced by the contribution of the off-diagonal bias $V_{g e}$. Due to the $\Theta$-function in Eq. 2, $V_{G}$ is constructed such that large values of $\Delta E_{\text {meta }}$ are biased and the metadynamics drives the system to the next intersection point. This requires that $V_{g e}$ is made dependent on an additional collective variable $s_{C I}$. If the latter is chosen in a way that it is able to distinguish between different molecular configurations, the return to the previously sampled regions of the CI seam is prevented. Complementary to $V_{G}, V_{g e}$ is updated only if the energy gap $\Delta E_{\text {meta }}$ is below $\epsilon$ by addition of a Gaussian potential according to

$$
\begin{aligned}
V_{g e}(t)= & \sum_{t^{\prime}=\tau_{G}, 2 \tau_{G}, \ldots}^{t} w \exp \left(-\frac{\left(s_{C I}(t)-s_{C I}\left(t^{\prime}\right)\right)^{2}}{2 \delta s^{2}}\right) \\
& \times \Theta\left(\epsilon-\Delta E_{\text {meta }}\left(t^{\prime}\right)\right) .
\end{aligned}
$$


As illustrated in Fig. 1a, $\Delta E_{\text {meta }}$ exactly equals the BO gap $\Delta E_{B O}$ as long as no Gaussians have been added to the off-diagonal bias $V_{g e}$, since $\mathbf{H}_{\text {meta }}$ reduces to $\mathbf{H}_{\mathbf{B O}}$. During the starting phase, $\Delta E_{\text {meta }}$ is by far larger than $\epsilon$, meaning that $V_{G}$ is periodically updated and drives the system toward the intersection seam. Only when $V_{G}$ is strong enough to persistently force the system to the vicinity of the CI seam, $V_{g e}$ is updated (see Fig. 1b). Diagonalization of $\mathbf{H}_{\text {meta }}$ enlarges the effective energy gap and the dynamics is continued on the modified PES. $V_{g e}$ generates a force to change the current value of the collective variable $s_{C I}$, while $V_{G}$ pushes the system back to the intersection seam. Upon reaching the next conical intersection, another Gaussian is added to $V_{g e}$ (see Fig. 1c), which is specifically adjusted to bias the current CI-structure. This process is repeated (see Fig. 1d) until the whole part of the intersection seam accessible by $s_{C I}$ is automatically "unzipped".

Besides the BO energy gradient of the ground state that can be obtained by a whole spectrum of electronic structure methods, the gradient of the modified energy gap is needed in order to calculate the force in Eq. 1. The latter can be obtained from the following expression:

$$
\nabla\left(\Delta E_{\text {meta }}\right)=\frac{\Delta E_{B O} \nabla\left(\Delta E_{B O}\right)+4 V_{g e} \nabla\left(V_{g e}\right)}{\Delta E_{\text {meta }}},
$$

which additionally requires the calculation of the excited state energy gradient, as well as differentiation of $V_{g e}$ with respect to the coordinates

$$
\begin{aligned}
\nabla\left(V_{g e}\right)= & \sum_{t^{\prime}=\tau_{G}, 2 \tau_{G}, \ldots}^{t} w \exp \left(-\frac{\left(s_{C I}(t)-s_{C I}\left(t^{\prime}\right)\right)^{2}}{2 \delta s^{2}}\right) \\
& \times \Theta\left(\epsilon-\Delta E_{\text {meta }}\left(t^{\prime}\right)\right) \\
& \times\left(-\frac{s_{C I}(t)-s_{C I}\left(t^{\prime}\right)}{\delta s^{2}} \nabla\left(s_{C I}(t)\right)\right) .
\end{aligned}
$$

The algorithm as implemented in the metaFALCON package is summarized in the flowchart given in Fig. 2. It consists of a standard MD code where the forces used for the integration of the Newtonian equations of motion are modified as described above. A key feature of our 


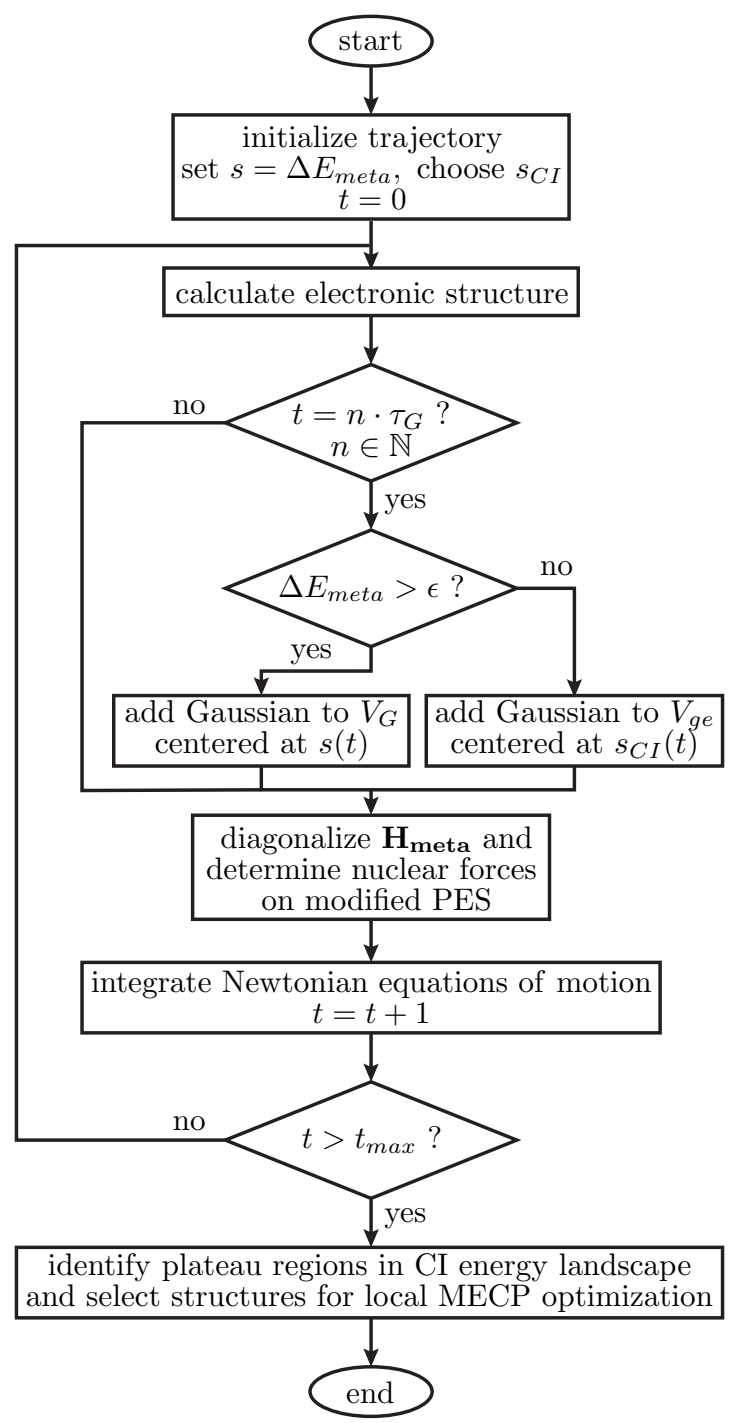

Figure 2: Algorithm flowchart of the multistate metadynamics. After choosing an appropriate $\mathrm{CV}$ for the off-diagonal bias potential, a conventional molecular dynamics algorithm is used for integrating the Newtonian equations of motion on the PES modified by $V_{G}$. Finally, local MECP optimizations are carried out for the characterization of the CI landscape. 
algorithm is the threshold $\epsilon$ in Eq. 2 and 5 that controls which bias potential is updated in a given metadynamics step. $V_{g e}$ is only updated, when $\Delta E_{m e t a}$ is small so that it acts only if the system is close to the CI seam. The complementary addition of Gaussians to $V_{G}$ only when $\Delta E_{\text {meta }}$ is large ensures that there is a force leading into the direction of the intersection seam at all times. We therefore recommend to set $\epsilon$ to the same value that is used for the parameter $w$ in Eq. 2. Plateau regions in the electronic BO energies then correspond to the CI energy landscape if $\Delta E_{B O}$ is low. Sampling from these periods of the dynamics allows for local CI optimization and subsequent classification of the found MECPs by structure and energy.

The choice of the collective variable $s_{C I}$. For the determination of the metadynamics forces, it is necessary to calculate $s_{C I}$ and its gradient in every dynamics step. Ideally, $s_{C I}$ should be unique for any structure on the intersection seam, which would be the case for example for the entries of the geometrical distance matrix. Unfortunately, an efficient metadynamics sampling of the configuration space is only achieved if the number of CVs is kept low which is definitely not the case for the complete distance matrix. Apart from that, symmetry ambiguities arise from the fact that the atoms have to be in a pre-defined order and commutation leads to a new matrix although the overall molecular shape is preserved. For these reasons, a better choice is to use scalar invariants like its lowest eigenvalue or other topological indices. ${ }^{38}$ As a most general approach in our simulations, we choose the 3D-Wiener number $W^{39}$ defined as

$$
W=\frac{1}{2} \sum_{i}^{N} \sum_{j}^{N} d_{i j}
$$

as a $\mathrm{CV}$ for the off-diagonal bias $s_{C I}$, where $N$ is the number of atoms and $d_{i j}$ are interatomic distances. It is convenient to consider only non-hydrogen distances, but we also examine the Wiener number including distances from and to hydrogen atoms, which we denote $W^{H}$. $W$ correlates with the molecular shape and therefore enables the efficient sampling of a large 
variety of different configurations for a given system. Due to the reduction of dimensionality, there is no unique mapping of the Wiener number to the structure, meaning that $f-3$ dimensional isosurfaces are biased rather than single configurations on the $f$-2-dimensional CI seam, where $f$ is the number of internal degrees of freedom. However, this problem can be adressed by running several trajectories with different initial conditions, leading to the largest possible structural variability.

The gradient of the collective variable, $\nabla\left(s_{C I}(t)\right)$, is needed for the evaluation of $\nabla\left(V_{g e}\right)$ according to Eq. 7 and for the 3D-Wiener number reads

$$
\nabla W=\frac{1}{2} \sum_{i}^{N} \sum_{j}^{N} \nabla d_{i j} .
$$

In aromatic and heteroaromatic organic molecules, many structures corresponding to conical intersections involve the displacement of atoms out of the ideal ring plane (ring puckering). We have already shown for the furan molecule ${ }^{29}$ that it is possible to find some of these structures by the use of the Wiener number. However, distinction between different puckering motifs can only be achieved if a suitable collective variable is employed that is able to characterize the exact type of structural deformation. Therefore, we have chosen the systematics of Cremer-Pople ring puckering parameters which allow for the full description of puckering in six-membered rings with only three coordinates. ${ }^{40}$ For a detailed description of the definition of Cremer-Pople parameters and the corresponding gradients see appendix A.

\section{Computational details}

The multistate metadynamics simulations on 1,3-butadiene and benzene have been performed by using the ab initio complete active space self-consistent field (CASSCF) method as implemented in the MoLPRo 2012 program package. ${ }^{41}$ The active space comprised all $\pi$-orbitals with the respective number of electrons, i.e. $(4,4)$ for butadiene and $(6,6)$ for 
benzene, and state-averaging has been applied for the lowest two singlet states. We have used the $4-31 \mathrm{G}$ basis set ${ }^{42}$ on butadiene and the $6-31 \mathrm{G}^{*}$ basis set ${ }^{43}$ on benzene. For the 9H-adenine molecule, we employed the semiempirical OM2 Hamiltonian combined with the GUGA MR-CI approach, ${ }^{44,45}$ taking into account all single and double excitations of the four most important reference configurations. The computational parameters were chosen in order to ensure comparability with previous results from the literature. ${ }^{15,31,46}$

In order to perform multistate metadynamics, ten initial conditions were generated for each of the molecules by sampling over 2 ps trajectories propagated in the electronic ground state. The Newtonian equations of motion were integrated using the velocity Verlet algorithm ${ }^{47}$ with a time step of $0.25 \mathrm{fs}$ (butadiene and benzene) and $0.1 \mathrm{fs}$ (adenine). Temperature was kept constant at $300 \mathrm{~K}$ using the Berendsen thermostat. ${ }^{48}$ The parameters for multistate metadynamics were chosen such that the intersection seam is reached within short simulation times. Correspondingly, Gaussians with a width of $0.5 \mathrm{eV}$ and a height of $1.0 \mathrm{eV}$ were added to $V_{G}$, while $\tau_{G}$ was set to 100 time steps $(25 \mathrm{fs}$ ). In the case of adenine, smoother sampling was achieved by adding Gaussians with a height of only $0.2 \mathrm{eV}$ in intervals of 250 time steps. According to the chosen $\delta s$, the threshold $\epsilon$ for the $\Theta$-functions in Eqs. 2 and 5 was also set to $0.5 \mathrm{eV}$. The shape of the Gaussians added to $V_{g e}$ has been adjusted to the respective CVs and is discussed in detail in section 4.1.

\section{Results and Discussion}

\section{$4.1 \quad 1,3$-butadiene}

As a first example we apply our method to explore the conical intersection seam in 1,3butadiene. In the past decades, the role of conical intersections in the photochemistry of butadiene has been extensively studied as a model for conjugated $\pi$-systems and photoisomerizations. ${ }^{31-33}$ The multistate metadynamics trajectory has been started from the ground state equilibrium structure with the Franck-Condon excitation energy of $6.65 \mathrm{eV}$. The inser- 
tion of several Gaussians to the bias potential already induces oscillations in $\Delta E_{\text {meta }}$ between $4 \mathrm{eV}$ and $10 \mathrm{eV}$. The pathway from the ground state minimum structure to the intersection seam is represented by the time evolution of the energy gap $\Delta E_{\text {meta }}$, depicted in Fig. 3a. Since $V_{g e}$ is zero in the displayed time range, $\Delta E_{\text {meta }}$ equals $\Delta E_{B O}$. As can be seen, the energy gap decreases within 600 fs from $6.65 \mathrm{eV}$ to zero, which reflects the fact that the trajectory reaches the intersection point between the ground and excited state for the first time.

The shape of the metadynamics potential $V_{G}$ is depicted in Fig. 3b in steps of five Gaussians $(0.125 \mathrm{ps})$. Within the first $0.5 \mathrm{ps}, V_{G}$ is only slightly extended to the lower values of the gap. However, after 25 Gaussians have been added to $V_{G}(0.625 \mathrm{ps})$, the induced forces are strong enough to let $\Delta E_{\text {meta }}$ become smaller than the threshold $\epsilon$. Consequently, the $\Theta$-function in Eq. 2 stops the addition of further Gaussians and the potential $V_{G}$ does not change as long as the system stays in the vicinity of the intersection seam.

The efficiency of the CI seam exploration is dependent on the chosen collective variable $s_{C I}$ to build up $V_{g e}$ and the respective parameters for the Gaussian shape. The CV, on the one hand, can be essentially one of two types, either specific to a given problem or of most possible general nature. The form of the Gaussians, on the other hand, requires the critical choice of $\delta s$ and $w$ in a way that the simulation time can be kept low with a maximum number of found MECP structures. In order to test the sensitivity of the method with respect to the choice of the Gaussian parameters we have run simulations with different values of $\delta s$ and $w$. Since the role of conical intersections between ground and excited states of butadiene has first been discussed in 1993, ${ }^{31}$ more than 30 different MECP structures have been reported for this small molecule. ${ }^{34}$ Most importantly, s-transoid and s-cisoid structures have been described, differing in their rotation angles around the central $\mathrm{C}-\mathrm{C}$ bond. We therefore concentrate on these two MECPs by setting $s_{C I}$ to the torsion angle $\phi$ defined by the four carbon atoms.

A comparison of differently shaped Gaussians added to $V_{g e}$ when $\Delta E_{B O}$ reaches zero for 

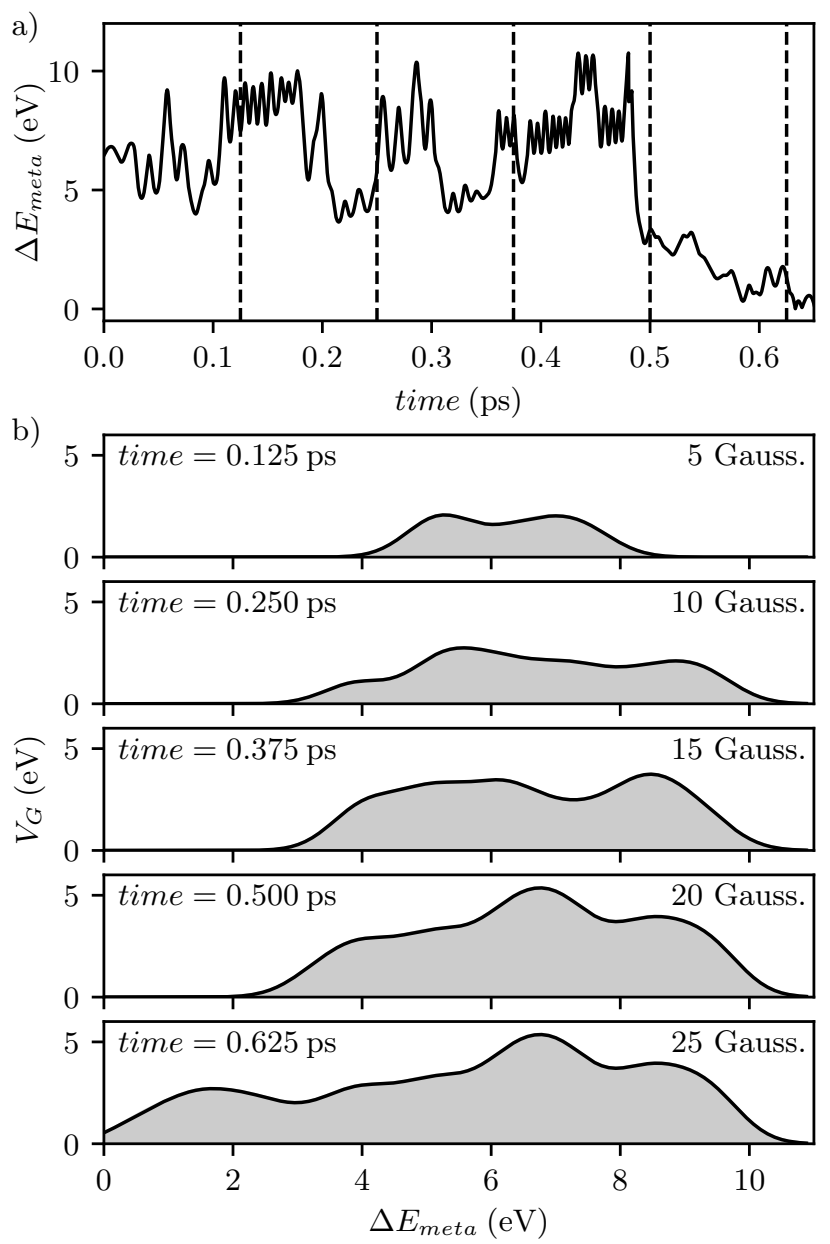

Figure 3: a) Energy gap $\Delta E_{\text {meta }}$ over time until the intersection seam is reached for the first time. b) Metadynamics potential $V_{G}$ dependent on the collective variable $\Delta E_{\text {meta }}$ at time steps symbolized by the dashed lines in a). The time steps are chosen corresponding to multiples of 5 Gaussians added to $V_{G}$. 

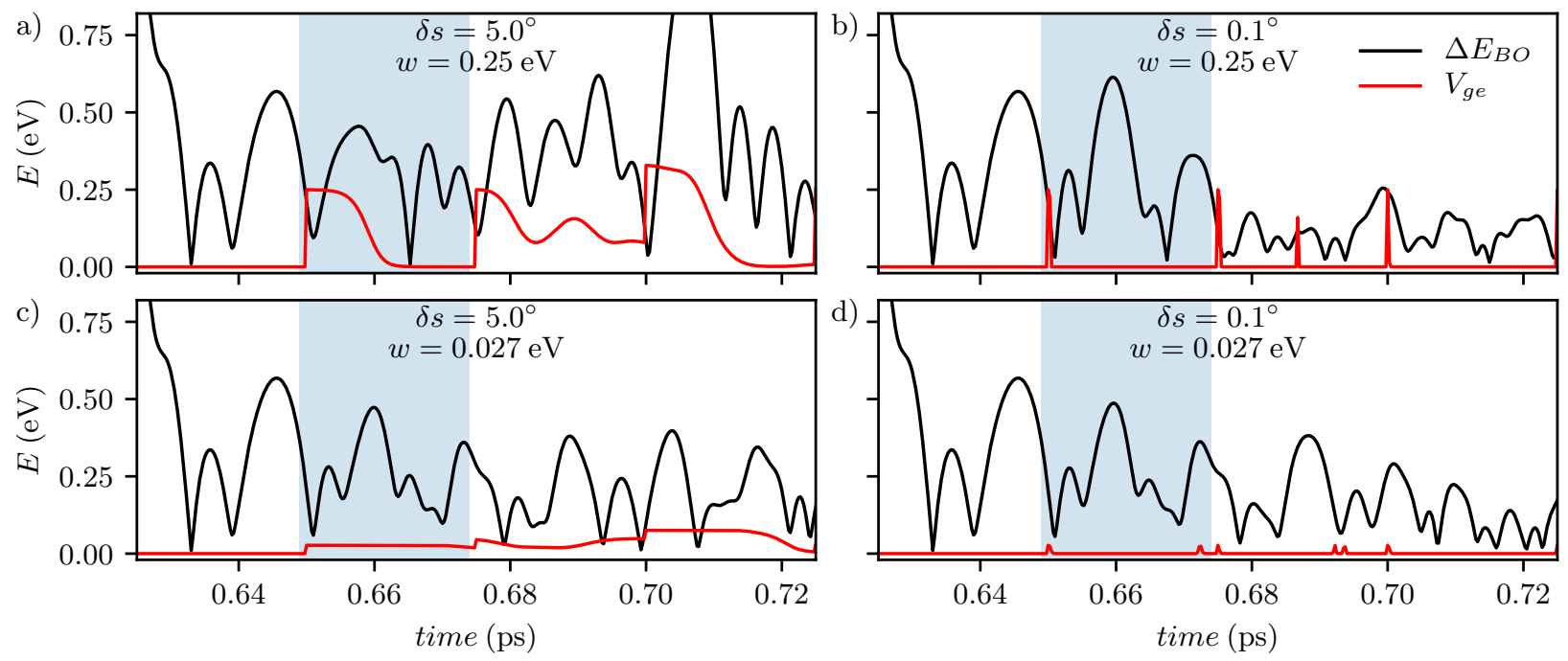

Figure 4: Comparison of trajectories with different Gaussian shapes in $V_{g e}$ for butadiene employing the torsion angle $\phi$ as the CV. The Born Oppenheimer energy gap $\Delta E_{B O}$ (black) and the off-diagonal coupling $V_{g e}$ (red) are given for a) broad $\delta s$ and high $w$, b) broad $\delta s$ and low $w, \mathrm{c}$ ) narrow $\delta s$ and high $w$ and d) narrow $\delta s$ and low $w$. The $25 \mathrm{fs}$ of relaxation subsequent to the insertion of the first Gaussian are highlighted in light blue.

the first time is provided in Fig. 4. In all of the four distinct combinations of $w$ and $\delta s$, the trajectory follows the same pathway until the addition of the first Gaussian to $V_{g e}$ at 0.65 ps. When Gaussian heights are of the same order of magnitude as $\Delta E_{B O}$ and large values for the width $\delta s$ are used, the algorithm can be nicely tracked step by step (see Fig. 4a). After the first occurrence of a non-zero $V_{g e}$, the forces resulting from $V_{G}$ provoke a reduction of the latter which is related to a changing torsion angle $\phi$. This process is completed at $0.67 \mathrm{ps}$, right before the addition of the second Gaussian. If the system returns to a point that has been biased before, $V_{g e}$ increases again, as is seen around 0.69 ps. However, these parameters correspond to a relatively coarse-grained intersection seam following, since permanently high values of $V_{g e}$ support the increase of $\Delta E_{B O}$, meaning that the trajectory is allowed to temporarily sheer off from the intersection seam. Therefore, it is desirable to reduce both parameters to a minimum in order to stay in closest vicinity to the crossing region. If only the width is decreased (see Fig. 4b), the result is an off-diagonal potential $V_{g e}$ composed from very narrow Gaussians with large derivatives close to their maxima, leading 
to an overshooting of the metadynamics forces. If instead only the height is decreased (see Fig. 4c), the system stays on the plateau-like top of the Gaussians for long times, because the acting force is negligible. The best performance is therefore achieved by choosing small values of $w$ and $\delta s$ simultaneously (see Fig. $4 \mathrm{~d}$ ). In the present paper, we use values of $0.1^{\circ}$ for $\delta s$ and $0.027 \mathrm{eV}$ for $w$.

In general, $V_{g e}$ is less sensitive to the choice of $\delta s$ and $w$ than $V_{G}$ in conventional metadynamics, if the multistate metadynamics is only used to localize as many CI structures as possible. However, if the aim is to achieve convergence to an intersection seam hypersurface as described in section 4.3, the guidelines known from the traditional metadynamics apply. ${ }^{49}$
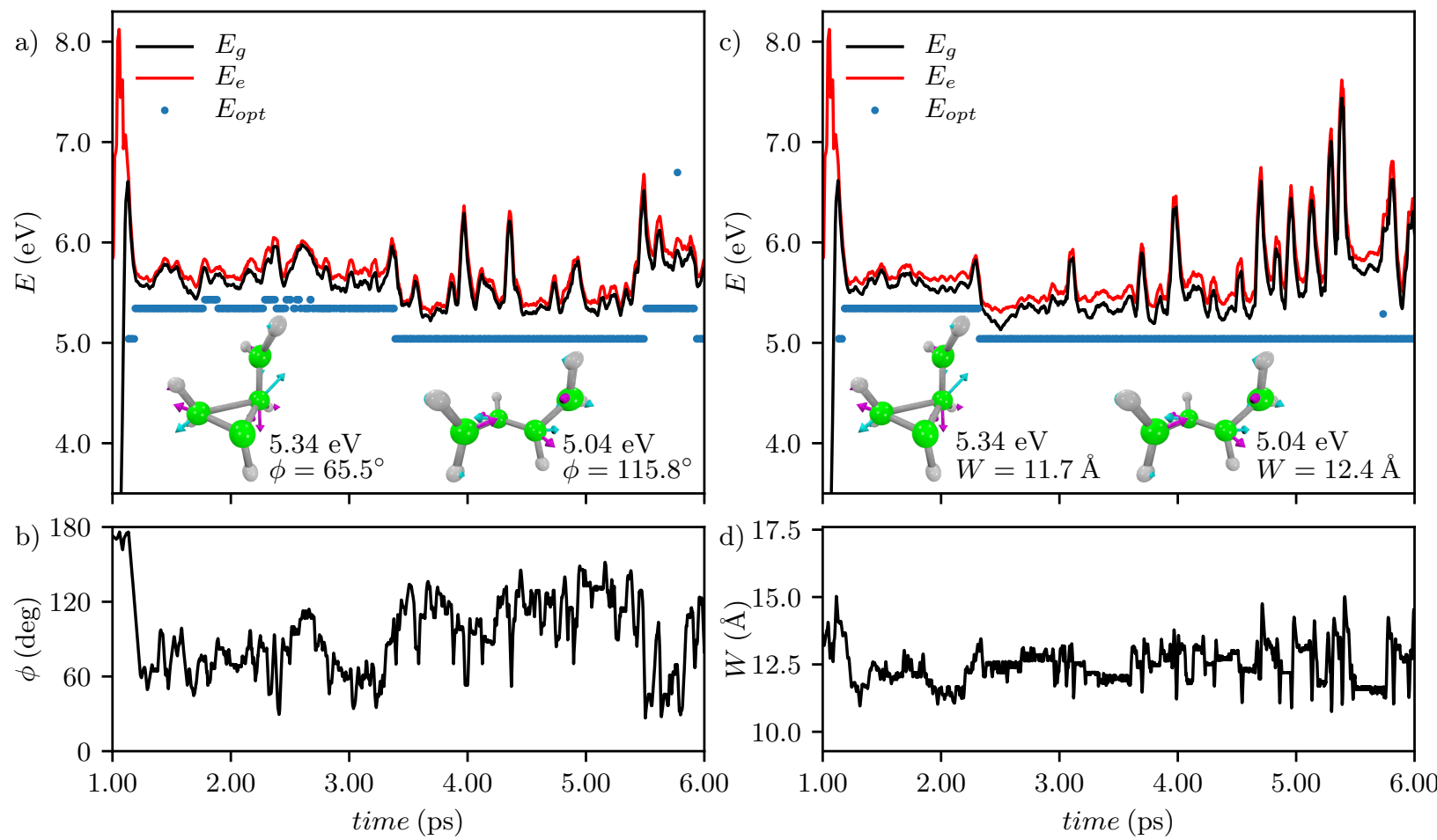

Figure 5: a) Ground and excited state energies, as well as energies of optimized snapshots for an example trajectory of 1,3-butadiene using the torsion angle $\phi$ as CV. The insets show the most important optimized MECP structures together with their gradient difference and non-adiabatic coupling vectors plotted as arrows. The corresponding $\mathrm{CV} \phi$ is depicted in b). c) Ground and excited state energies, as well as energies of optimized snapshots for an example trajectory of 1,3-butadiene with the same initial conditions as in a) but using the Wiener number $W$ as CV. Again, the insets show the most important optimized MECP structures and in d) the corresponding $\mathrm{CV} W$ is depicted. 
As has been pointed out before, $\phi$ is the most natural choice of $s$ for the representation of the well-characterized s-cisoid and s-transoid MECP structures of butadiene. The ground and excited state energies of an exemplary trajectory using this CV are depicted in Fig. 5a. The overall shape of $E_{g}$ and $E_{e}$ over time follows the energies of sampled snapshot geometries that are obtained by local optimization using the Bearpark-Robb optimization scheme for MECPs. ${ }^{13}$ When the intersection seam is reached after the first $1.2 \mathrm{ps}$ of the simulation, $\phi$ (see Fig. 5b) already takes values close to the torsion angle of $65.5^{\circ}$ that is obtained in optimized structures of the s-cisoid MECP $(5.34 \mathrm{eV})$ and oscillates around this angle for the next 2 ps. However, in the regions where $\phi$ takes larger values, optimization of snapshots converge to a local minimum with similar structure compared to the lowest s-cisoid structure with a relative energy of $5.43 \mathrm{eV}$ and $\phi=69.9$. At $3.3 \mathrm{ps}$, another plateau is reached at lower energy, associated to the optimized s-transoid structure with a torsion angle of $115.8^{\circ}$ and a relative energy of $5.04 \mathrm{eV}$.

The drawback of using a specialized CV like the torsion angle for butadiene is that it requires some preliminary knowledge on the character of existing CI-structures. For this reason, we have already shown for the furan molecule that the $3 \mathrm{D}$ Wiener number $W$ is a suitable choice of a generalized CV for multistate metadynamics. ${ }^{29}$ Here, we prove that $W$ is also appropriate for butadiene by comparison with the results obtained using the torsion angle. We used a Gaussian width $\delta s$ of $0.1 \AA$ and $w$ was set to $0.027 \mathrm{eV}$ as in the previous example.

In the trajectory given in Fig. 5c, most of the optimized structures found are the same as in Fig. 5a. The energy pathway in the beginning after closing the gap $\Delta E_{\text {meta }}$ is equal to the torsion angle $\mathrm{CV}$, since the forces resulting from $V_{G}$ need some time to manipulate the dynamics. Hence, the structure in this time range is of s-cisoid character, leading to a Wiener number of $11.7 \AA$ upon MECP optimization of dynamics snapshots. Then, with much shorter time delay compared to the usage of the torsion angle, the transition to the s-transoid structure is observed. The latter exhibits a Wiener number of $12.4 \AA$ and can 
therefore reduce the off-diagonal coupling acting as a bias for low $W$ at that time. It is worth to note that in contrast to the application of the torsion angle as $\mathrm{CV}$, the trajectory does not return to the s-cisoid structure within the given time range. The reason for that is that values for $W$ are not unique with respect to different structures. From 5 ps onwards, the Wiener number repeatedly gets similar to that of the s-cisoid structure although the overall character is still s-transoid. As a consequence, there is no force that induces a change back to the original torsion angle.

\subsection{Benzene}

In this section we discuss multistate metadynamics simulations on benzene, which serves as a representative of the class of aromatic molecules. It exhibits a large number of MECPs between the electronic ground and excited states, ${ }^{15}$ of which a half-boat shaped ring-puckering structure is the best known one. ${ }^{35}$

Interestingly, a number of differing MECP structures have been reported with similar geometrical arrangement of the carbon atoms, i.e. the positions of hydrogen atoms are crucial to distinguish between them. ${ }^{15}$ Especially ring-puckering structures are typically found in the low-energy regions of the intersection seam. For this purpose, we tested the use of the Wiener number $W^{H}$ including all distances to hydrogen atoms and compared the results to these obtained with the Wiener number $W$ excluding hydrogens. Selected optimized structures and their corresponding $W$ and $W^{H}$ values are presented in Fig. 6. In the metadynamics simulations we used values of $0.027 \mathrm{eV}$ and $0.01 \AA$ for the $w$ and $\delta s$ parameters, respectively. In order to account for the larger changes in the values of $W^{H}$ compared to $W$, $\delta s$ was increased to $0.05 \AA$, when hydrogen atoms were taken into account. The simulations show that both collective variables are appropriate for the efficient localization of the low-energetic and most important ring-puckering structures at $5.49 \mathrm{eV}$ and $5.76 \mathrm{eV}$. In most trajectories, the intersection seam is first reached close to the higher energy structure and after a short time, relaxation to the lower-energetic one with the hydrogen atom in equatorial position takes 


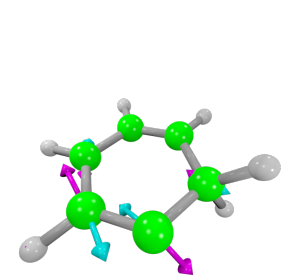

$4.88 \mathrm{eV}$ $32.0 \AA / 181.5 \AA$

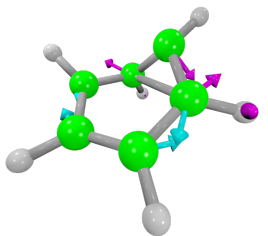

$5.87 \mathrm{eV}$ $31.0 \AA / 177.8 \AA$

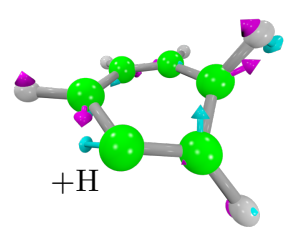

$7.96 \mathrm{eV}$ $31.4 \AA / 203.1 \AA$

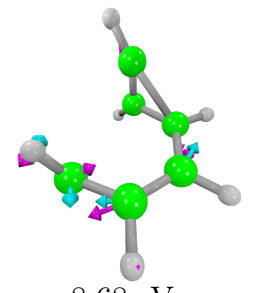

$8.68 \mathrm{eV}$ $35.5 \AA / 191.4 \AA$

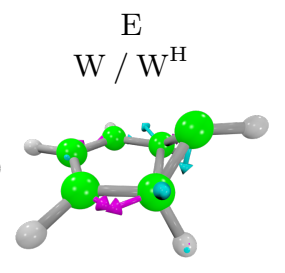

$5.49 \mathrm{eV}$ $30.9 \AA / 179.7 \AA$

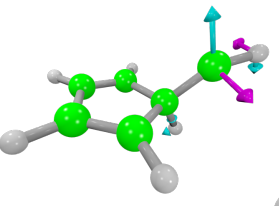

$6.42 \mathrm{eV}$ $32.4 \AA / 185.0 \AA$

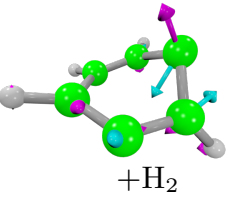

$8.64 \mathrm{eV}$ $30.8 \AA / 255.9 \AA$

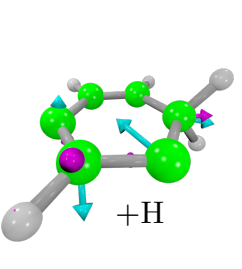

$8.83 \mathrm{eV}$ $32.0 \AA / 200.5 \AA$

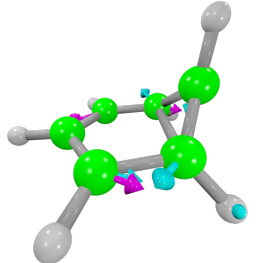

$5.76 \mathrm{eV}$

$31.2 \AA / 179.4 \AA$

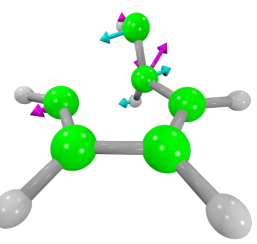

$7.35 \mathrm{eV}$

$34.2 \AA / 189.3 \AA$

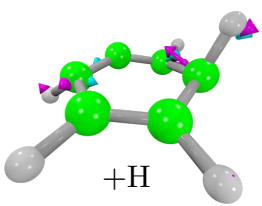

$8.68 \mathrm{eV}$
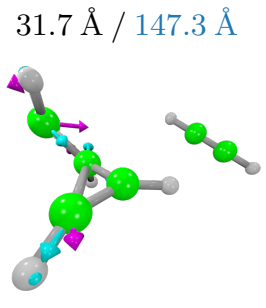

$10.03 \mathrm{eV}$ $50.5 \AA / 234.0 \AA$

Figure 6: Optimized MECP structures of benzene obtained with multistate metadynamics followed by local MECP optimization. The gradient difference and non-adiabatic coupling vectors are displayed as arrows. Energies are given relative to the optimized ground state structure and the Wiener number is provided by calculation with and without consideration of hydrogen atoms. Numbers highlighted in blue mean that the structure has been obtained by application of the respective CV. 
place. A similar structure with reduced symmetry is found at $5.87 \mathrm{eV}$ in some trajectories. Since the Wiener number has a natural lower bound, long simulation times usually lead to increasing values. Therefore, compared to $W^{H}$ the use of $W$ excluding hydrogens tends to favor geometrical rearrangements and fragmentations of the carbon scaffold at longer times. In the example of benzene, 5-membered ring structures and ring-openings are found in the range above $6.42 \mathrm{eV}$. Another frequently observed channel leading to the damage of benzene is the dissociation of an acetylene unit.

Since the Wiener number is defined as the sum over all interatomic distances, its value is larger the more atoms are included. Furthermore, non-bonding $\mathrm{H}-\mathrm{H}$ and $\mathrm{C}-\mathrm{H}$ distances are generally larger than $\mathrm{C}-\mathrm{C}$ distances, since hydrogen atoms are located at the outside of the benzene ring. For this reason, the impact of $\mathrm{C}-\mathrm{C}$ distances on changes in $W^{H}$ is generally lower than for distances including hydrogen atoms. Forces to change the Wiener number may therefore lead to irreversibly large displacements of hydrogen atoms, while more subtle changes in the geometry can be overlooked. The ensemble of higher-energetic MECP structures obtained with the hydrogen-including $W^{H}$ hence contains mainly modifications of ring-puckering structures with dissociated hydrogen atoms. However, the application of $W^{H}$ also enabled the characterization of a planar ring structure (see first structure in Fig. 6) generated by hydrogen transfer of two neighboring carbon atoms that, interestingly, lies more than $0.6 \mathrm{eV}$ below the equatorial ring-puckering (see second structure in Fig. 6).

\subsection{H-adenine}

The previous sections have demonstrated that the outcome of the multistate metadynamics is mainly dependent on which collective variable is employed. The metadynamics method, however, also allows one to use multiple CVs, if the Gaussian functions in Eq. 5 are re-

placed by products of Gaussians with different $s_{C I}$. If a set of CVs is defined such that it approximately spans the branching plane perpendicular to the intersection seam, the negative multistate metadynamics potential can be used to reconstruct the intersection seam 
hypersurface just like standard metadynamics yields the free energy surface. This will be illustrated here on the example of $9 \mathrm{H}$-adenine. It is worth noting that nonradiative decay upon photoexcitation does not need to proceed through a MECP but can also be probable from higher energy regions, depending on the magnitude and direction of nuclear velocities. Since the obtained hypersurface is spanned over the complete CV space, it is a more general representation of the CI seam than methods which rely only on MECP optimization and connecting pathways. DNA nucleobases are a paramount example of molecules whose deactivation processes proceed through conical intersections, which prohibits their damage upon UV-excitation. ${ }^{7}$ The key MECPs found in these molecules are characterized by heteroaromatic ring-puckering structures. For example, in the case of adenine, a great number of ring-puckering MECPs has been found and characterized, ${ }^{50}$ most of them lying more than $5 \mathrm{eV}$ above the ground state minimum. However, deformation of the six-membered pyrimidine ring system leads to the four low-energetic MECPs $\mathbf{1 - 4},{ }^{51}$ with the $\mathrm{NH}_{2}$-group or the $\mathrm{H}$-atom arranged nearly $90^{\circ}$ with respect to the ring plane, respectively (cf. Fig. 7a). Such ring-puckering structures can be characterized and distinguished by the Cremer-Pople parameters ${ }^{40}$ that allow the precise characterization of six-membered rings with only three variables. Besides an amplitude $Q$, two angles $\phi$ and $\theta$ are calculated from the displacement of the ring atoms out of the plane, as described in detail in the Appendix A. Their large flexibility paired with structural unambiguity makes Cremer-Pople parameters the perfect CVs for investigating the conical intersection seam of cyclic structures such as adenine. Such multiple CVs for the construction of $V_{g e}$ in multistate metadynamics can be implemented analogously as in conventional metadynamics. Here, we use the multistate metadynamics with $Q, \phi$ and $\theta$ as CVs aiming to obtain a complete picture of the intersection seam in adenine with respect to ring-puckerings in the pyrimidine unit.

The exemplary multistate metadynamics trajectory for adenine shown in Fig. 8 leads to the formation of three out of four expected CI structures. As can be seen, starting from the ground state structure, after $1.5 \mathrm{ps}, \Delta E_{\text {meta }}$ drops close to zero and the intersection seam is 
a)

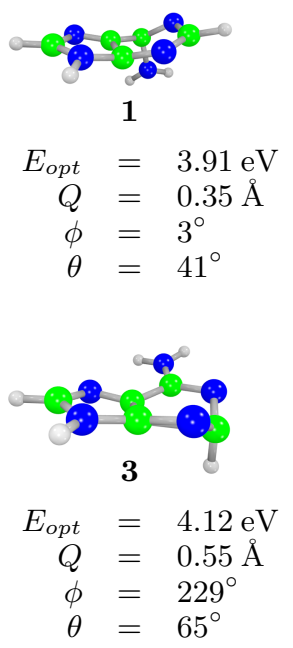

b)
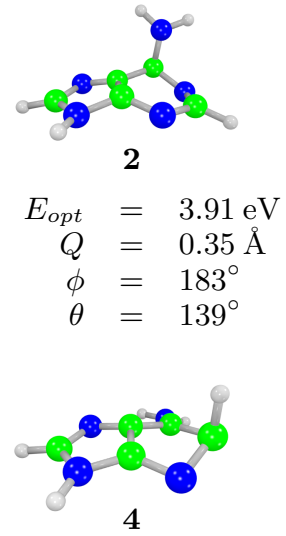

$E_{\text {opt }}=4.12 \mathrm{eV}$

$Q=0.55 \AA$

$\phi=49^{\circ}$

$\theta=115^{\circ}$

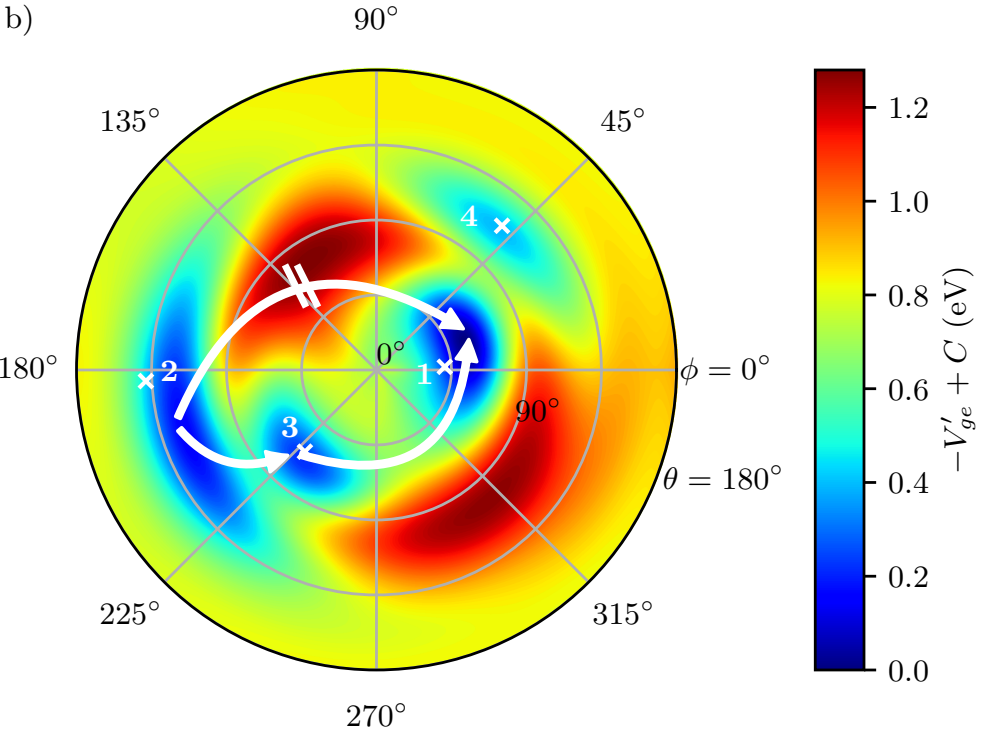

Figure 7: a) Literature-known optimized MECP structures of adenine 1 to 4 together with their energies referenced to the ground state equilibrium and Cremer-Pople parameters. b) Relative intersection seam hypersurface obtained with multistate metadynamics using Cremer-Pople parameters as CVs. The MECPs are marked with white crosses and the white arrows symbolize MECP-connecting pathways on the surface. Energies have been shifted by a constant $C$ such that the lowest MECP 1 lies at $0 \mathrm{eV}$.

reached through a $\mathrm{NH}_{2}$-puckered structure that can be assigned to structure $\mathbf{2}$ in Fig. 7a. Shortly afterwards, the gap increases again, but the trajectory returns to the intersection seam and stays on the higher-lying plateau corresponding to the structure 3 for $0.7 \mathrm{ps}$. Finally, the energy is decreased again, leading to structure $\mathbf{1}$ which is similar to $\mathbf{3}$.

A pitfall of using Cremer-Pople parameters as CVs again are dissociation events of hydrogen atoms that can occur during the simulation. Since ring-puckering is coupled to large values of $Q$, reducing the amplitude provides an escape pathway from the already built up bias potential $V_{g e}$. Low values of $Q$ however correspond to a flattening of the ring structure that usually would cause an increase of $\Delta E_{\text {meta }}$. The dissociation of a hydrogen atom is the most probable pathway in such a situation, since this leads to a decreasing energy gap also for a planar ring structure. Despite the fact that ultrafast hydrogen abstractions are physically meaningful pathways, ${ }^{51}$ they cause an irreversible structure damage. For that reason, it is unfortunately not possible to drive a single trajectory until the convergence of the 

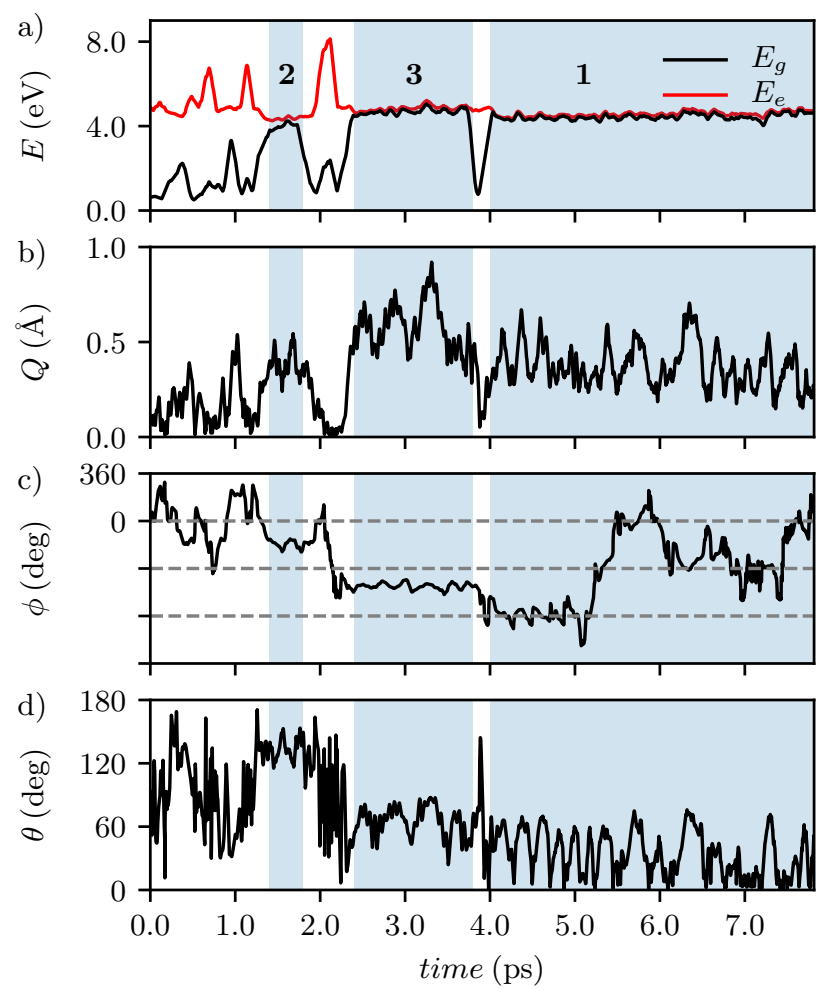

Figure 8: Example trajectory of multistate metadynamics of adenine. Besides the a) ground and excited state energies along the trajectory, b) the amplitude $Q$, c) the angle $\phi$ and d) the angle $\theta$ are depicted. The bold numbers in a) correspond to the structure character in the surrounding blue-highlighted time ranges. Note that $\phi$ is periodic in the range from $0^{\circ}$ to $360^{\circ}$ which is illustrated by horizontal dashed lines in c). 
metadynamics potential because the failure of the semiempirical SCF calculation forces the simulation run to stop whenever a dissociation event occurs. Therefore, we ran multiple trajectories with different initial conditions and, inspired by multiple walkers metadynamics, ${ }^{25}$ summed up the individually obtained $V_{g e}$ potentials to a single potential $V_{g e}^{\prime}$. The number of trajectories has been gradually increased until the approximate convergence of $V_{g e}^{\prime}$ was achieved. Since $Q$ is only the amplitude of the puckering processes and does not include information on the type of puckering, it was integrated out of the potential in the range from $0.13 \AA$ to $1.06 \AA$ for the sake of visualization. The resulting total potential from 20 trajectories depends only on $\phi$ and $\theta$. It exhibits four maxima that can be assigned to the minimum energy crossing points $\mathbf{1 - 4}$ on the intersection seam hypersurface which is depicted in Fig. $7 \mathrm{~b}$ as $-V_{g e}^{\prime}$ shifted by a constant $C$. As expected, the $\mathrm{NH}_{2}$-puckered structures are found to be more stable than the H-puckered ones. An average difference of $0.24 \mathrm{eV}$ is obtained from the multistate metadynamics simulation which nicely resembles the energy difference of $0.22 \mathrm{eV}$ between optimized $\mathbf{1} / \mathbf{2}$ and $\mathbf{3} / \mathbf{4}$ structures. The pathway that is followed by the trajectory in Fig. 8 can also be explained by the obtained intersection seam hypersurface. The direct conversion from 2 to 1 is extremely unfavorable (crossed white arrow in Fig. 7b) due to the high barrier $>1 \mathrm{eV}$. The two-step pathway observed in the example trajectory (consecutive white arrows), on the other hand, only requires the crossing of two relatively low barriers from 2 to $\mathbf{3}(0.36 \mathrm{eV})$ and from $\mathbf{3}$ to $\mathbf{1}(0.47 \mathrm{eV})$.

\section{Conclusion}

A multistate metadynamics algorithm has been developed and implemented into the metaFALCON program package allowing for automatic exploration of conical intersection seams between adiabatic Born Oppenheimer potential energy surfaces. In order to confine the MD simulation to the CI seam, the multistate electronic Hamiltonian is extended by introducing Gaussian-shaped off-diagonal bias potentials. By diagonalization of such an extended elec- 
tronic Hamiltonian and introduction of the energy gap between two states as a collective variable, a modified PES is obtained that can be used to run molecular dynamics within the CI seam. The algorithm can be straightforwardly combined with any ab initio or semiempirical electronic structure method that can provide ground and excited state energies and their gradients.

As an illustration, multistate metadynamics has been applied to explore the CI seams in 1,3-butadiene, benzene and $9 \mathrm{H}$-adenine, and the influence of the simulation parameters on the seam exploration has been systematically investigated. Starting from the ground-state minimum structures, we have performed simulations that show that the intersection seam in all three systems can be efficiently reached. Special attention has been paid to the choice of parameters that define the shape of the Gaussians contributing to the bias potential that is included in the multistate electronic Hamiltonian. Furthermore, the problem of finding the correct collective variable that drives the seam exploration has been addressed on the example of 1,3-butadiene. The use of the Wiener number has proven to successfully provide a reliable map of the conical intersection landscape. The impact of including hydrogen atoms into the calculation of the Wiener number has also been evaluated on the example of the benzene molecule. Both variants allowed us to systematically identify a large number of minimum energy crossing points, so we recommend using the Wiener number excluding hydrogen atoms as the most general starting point for any system. However, depending on the investigated system, a more specialized collective variable may be more appropriate in order to find all crossing points that may be relevant for understanding photochemical reaction pathways and nonradiative relaxation channels in complex molecular systems. Finally, on the example of $9 \mathrm{H}$-adenine we have demonstrated that multistate metadynamics can be used to obtain a global representation of the CI seam by converging the multistate metadynamics potential to a stable energy hypersurface. The developed methodology has been implemented into the program package metaFALCON that is publicly available free of charge (https://metafalcon.chemie.uni-wuerzburg.de/). 


\section{A Definition of Cremer-Pople parameters}

Cremer-Pople parameters as CVs in $N$-membered cyclic systems. In order to define the Cremer-Pople parameters in a $N$-membered cyclic system, it is first necessary to shift the molecular coordinates $\mathbf{R}$ with respect to the origin such that the following condition is satisfied,

$$
\sum_{i=1}^{N} \mathbf{R}_{\mathbf{i}}=0
$$

and rotate them in a way that the $x y$-plane is the mean ring plane and the atomic positions on the $z$-axis correspond to the displacements from the latter. In order to define such a plane uniquely, the $y$-axis is fixed to the position vector of atom 1 and two vectors

$$
\begin{aligned}
& \mathbf{R}^{\prime}=\sum_{i=1}^{N} \mathbf{R}_{\mathbf{i}} \sin [2 \pi(i-1) / N], \\
& \mathbf{R}^{\prime \prime}=\sum_{i=1}^{N} \mathbf{R}_{\mathbf{i}} \cos [2 \pi(i-1) / N]
\end{aligned}
$$

are used to define the unit normal vector of the plane

$$
\mathbf{n}=\frac{\mathbf{R}^{\prime} \times \mathbf{R}^{\prime \prime}}{\left|\mathbf{R}^{\prime} \times \mathbf{R}^{\prime \prime}\right|}
$$

Note that $\mathbf{R}^{\prime}$ and $\mathbf{R}^{\prime \prime}$ are dependent on the atom-numbering. The new $z$-coordinates of all atoms within the ring are then obtained by the projection of $\mathbf{R}_{\mathbf{i}}$ on $\mathbf{n}$

$$
z_{i}=\mathbf{R}_{\mathbf{i}} \cdot \mathbf{n}
$$

Based on this set of transformed coordinates, Cremer and Pople define $(N-3) / 2$ amplitudephase pairs if $N$ is odd. Otherwise, $(N-4) / 2$ amplitude-phase pairs are obtained alongside another single amplitude. A six-membered ring, for example, leads to the definition of the 
amplitude $q_{2}$ and phase $\phi_{2}$ in the following manner:

$$
\begin{aligned}
& q_{2} \sin \phi_{2}=-\frac{1}{\sqrt{3}} \sum_{i=1}^{6} z_{i} \sin \left(\frac{2}{3} \pi(i-1)\right)=: A \\
& q_{2} \cos \phi_{2}=\frac{1}{\sqrt{3}} \sum_{i=1}^{6} z_{i} \cos \left(\frac{2}{3} \pi(i-1)\right)=: B
\end{aligned}
$$

The additional single puckering amplitude $q_{3}$ reads

$$
q_{3}=\frac{1}{\sqrt{6}} \sum_{i=1}^{6}(-1)^{i-1} z_{i}
$$

Since the physical meanings of $q_{2}$ and $q_{3}$ are not straightforward, it is convenient to transform the three parameters from Eqs. 15-17 into a set of spherical polar coordinates $Q$, $\phi$ and $\theta$. For this reason, $q_{2}$ and $q_{3}$ are expressed in polar coordinates

$$
\begin{aligned}
& q_{2}=Q \sin \theta, \\
& q_{3}=Q \cos \theta .
\end{aligned}
$$

That leads, on the one hand, to the definition of the total puckering amplitude

$$
Q=\left(q_{2}^{2}+q_{3}^{2}\right)^{1 / 2}=\left(\sum_{i=1}^{6} z_{i}^{2}\right)^{1 / 2}
$$

which is zero for the flat ring structure and increases for larger $\left|z_{i}\right|$ values. On the other hand, the corresponding angle

$$
\theta=\arctan \frac{q_{2}}{q_{3}}
$$

represents the puckering character, i.e. the transition from one chair $\left(0^{\circ}\right)$ over boat / skew- 
boat $\left(90^{\circ}\right)$ structures to the other chair form $\left(180^{\circ}\right)$. The third parameter

$$
\phi=\phi_{2}=\arctan \frac{A}{B}
$$

can be understood as a phase angle and takes values from $0^{\circ}$ to $360^{\circ}$.

According to the chain rule, gradients of Cremer-Pople parameters split into the differentiation with respect to the vector of $z_{i}$-components $\mathbf{z}$ and the derivative of the latter with respect to the cartesian coordinates $\mathbf{R}$

$$
\nabla Q=\frac{\partial Q}{\partial \mathbf{z}} \frac{\partial \mathbf{z}}{\partial \mathbf{R}}, \nabla \phi=\frac{\partial \phi}{\partial \mathbf{z}} \frac{\partial \mathbf{z}}{\partial \mathbf{R}}, \nabla \theta=\frac{\partial \theta}{\partial \mathbf{z}} \frac{\partial \mathbf{z}}{\partial \mathbf{R}}
$$

Since $Q$ is only dependent on the components of $\mathbf{z}$ directly according to Eq. 20, its derivative takes the simple form

$$
\frac{\partial Q}{\partial \mathbf{z}}=\frac{\partial}{\partial \mathbf{z}} \sqrt{\sum_{i=1}^{6} z_{i}^{2}}=\frac{\mathbf{z}}{Q} .
$$

The derivative of $\phi$ with respect to $\mathbf{z}$

$$
\frac{\partial \phi}{\partial \mathbf{z}}=\frac{B \frac{\partial A}{\partial \mathbf{z}}-A \frac{\partial B}{\partial \mathbf{z}}}{B^{2}\left(1+(A / B)^{2}\right)}
$$

follows from Eq. 22 and requires the additional differentiation of the term $A$ from Eq. 15

$$
\frac{\partial A}{\partial z_{i}}=\sin \left(\frac{2}{3} \pi(i-1)\right)
$$

and the term $B$ from Eq. 16

$$
\frac{\partial B}{\partial z_{i}}=\cos \left(\frac{2}{3} \pi(i-1)\right) .
$$

For the evaluation of $\nabla \theta$, either of Eq. 18, Eq. 19 and Eq. 21 could be used, but the easiest 
approach is reached from the expression in Eq. 19

$$
\frac{\partial \theta}{\partial \mathbf{z}}=\left(\left(\frac{q_{3}}{Q}\right)^{2}-1\right)^{-1 / 2} \frac{Q \frac{\partial q_{3}}{\partial \mathbf{z}}-q_{3} \frac{\partial Q}{\partial \mathbf{z}}}{Q^{2}}
$$

because $\partial Q / \partial \mathbf{z}$ is easily accessible from Eq. 24 and $\partial q_{3} / \partial z_{i}$ is derived from Eq. 17 as

$$
\frac{\partial q_{3}}{\partial z_{i}}=\frac{1}{\sqrt{6}}(-1)^{i-1}
$$

Finally, each component of $\mathbf{z}$ depends on $\mathbf{R}$ in $\mathbf{R}_{\mathbf{i}}$ and $\mathbf{n}$, so its derivative is given by

$$
\frac{\partial z_{i}}{\partial \mathbf{R}}=\frac{\partial \mathbf{R}_{\mathbf{i}}}{\partial \mathbf{R}} \cdot \mathbf{n}+\mathbf{R}_{\mathbf{i}} \cdot \frac{\partial \mathbf{n}}{\partial \mathbf{R}}
$$

where $\partial \mathbf{R}_{\mathbf{i}} / \partial \mathbf{R}$ is trivial and the gradient of the normed normal vector has the form

$$
\frac{\partial \mathbf{n}}{\partial \mathbf{R}}=\frac{\frac{\partial}{\partial \mathbf{R}}\left(\mathbf{R}^{\prime} \times \mathbf{R}^{\prime \prime}\right)\left|\mathbf{R}^{\prime} \times \mathbf{R}^{\prime \prime}\right|-\left(\mathbf{R}^{\prime} \times \mathbf{R}^{\prime \prime}\right) \frac{\partial}{\partial \mathbf{R}}\left|\mathbf{R}^{\prime} \times \mathbf{R}^{\prime \prime}\right|}{\left|\mathbf{R}^{\prime} \times \mathbf{R}^{\prime \prime}\right|^{2}} .
$$

This requires the differentiation of the cross product between the vectors $\mathbf{R}^{\prime}$ and $\mathbf{R}^{\prime \prime}$

$$
\frac{\partial}{\partial \mathbf{R}}\left(\mathbf{R}^{\prime} \times \mathbf{R}^{\prime \prime}\right)=\frac{\partial \mathbf{R}^{\prime}}{\partial \mathbf{R}} \times \mathbf{R}^{\prime \prime}+\mathbf{R}^{\prime} \times \frac{\partial \mathbf{R}^{\prime \prime}}{\partial \mathbf{R}},
$$

with the $3 \times 3$ components

$$
\frac{\partial \mathbf{R}^{\prime}}{\partial \mathbf{R}_{\mathbf{i}}}=\sin \left(\frac{1}{3} \pi(i-1)\right) \mathbf{I}
$$

and

$$
\frac{\partial \mathbf{R}^{\prime \prime}}{\partial \mathbf{R}_{\mathbf{i}}}=\cos \left(\frac{1}{3} \pi(i-1)\right) \mathbf{I},
$$

where $\mathbf{I}$ is the three-dimensional identity matrix. 


\section{Acknowledgement}

J.O.L. and R.M. are grateful to the Deutsche Forschungsgemeinschaft (DFG) for financial support through GRK2112: "Molecular Biradicals: Structure, Properties and Reactivity". M.I.S.R. and R.M. acknowledge funding from the European Research Council (ERC) Consolidator Grant DYNAMO (Grant No. 646737).

\section{References}

(1) Yarkony, D. R. Diabolical conical intersections. Rev. Mod. Phys. 1996, 68, 985-1013.

(2) Domcke, W., Yarkony, D. R., Köppel, H., Eds. Conical Intersections: Electronic Structure, Dynamics and Spectroscopy; Advanced Series in Physical Chemistry; World Scientific: Singapore, 2004.

(3) Domcke, W., Yarkony, D. R., Köppel, H., Eds. Conical Intersections: Theory, Computation and Experiment; Advanced Series in Physical Chemistry; World Scientific: Singapore, 2011.

(4) Robb, M. A. Theoretical Chemistry for Electronic Excited States; Theoretical and Computational Chemistry Series; Royal Society of Chemistry: Cambridge, 2018.

(5) Bonačić-Koutecký, V.; Koutecký, J.; Michl, J. Neutral and Charged Biradicals, Zwitterions, Funnels in $\mathrm{S}_{1}$, and Proton Translocation: Their Role in Photochemistry, Photophysics, and Vision. Angew. Chem. Int. Ed. 1987, 26, 170-189.

(6) Polli, D.; Altoè, P.; Weingart, O.; Spillane, K. M.; Manzoni, C.; Brida, D.; Tomasello, G.; Orlandi, G.; Kukura, P.; Mathies, R. A.; Garavelli, M.; Cerullo, G. Conical intersection dynamics of the primary photoisomerization event in vision. $\mathrm{Na}$ ture 2010, 467, 440-443. 
(7) Schultz, T.; Samoylova, E.; Radloff, W.; Hertel, I. V.; Sobolewski, A. L.; Domcke, W. Efficient Deactivation of a Model Base Pair via Excited-State Hydrogen Transfer. Science 2004, 306, 1765-1768.

(8) Bernardi, F.; Olivucci, M.; Robb, M. A. Potential energy surface crossings in organic photochemistry. Chem. Soc. Rev. 1996, 25, 321.

(9) Musser, A. J.; Liebel, M.; Schnedermann, C.; Wende, T.; Kehoe, T. B.; Rao, A.; Kukura, P. Evidence for conical intersection dynamics mediating ultrafast singlet exciton fission. Nat. Phys. 2015, 11, 352-357.

(10) Kowalewski, M.; Bennett, K.; Dorfman, K. E.; Mukamel, S. Catching Conical Intersections in the Act: Monitoring Transient Electronic Coherences by Attosecond Stimulated X-Ray Raman Signals. Phys. Rev. Lett. 2015, 115, 193003.

(11) Adachi, S.; Schatteburg, T.; Humeniuk, A.; Mitrić, R.; Suzuki, T. Probing ultrafast dynamics during and after passing through conical intersections. Phys. Chem. Chem. Phys. 2019, doi: 10.1039/c8cp04426k.

(12) Ciminelli, C.; Granucci, G.; Persico, M. The Photoisomerization Mechanism of Azobenzene: A Semiclassical Simulation of Nonadiabatic Dynamics. Chem. Eur. J. 2004, 10, $2327-2341$.

(13) Bearpark, M. J.; Robb, M. A.; Schlegel, H. B. A direct method for the location of the lowest energy point on a potential surface crossing. Chem. Phys. Lett. 1994, 223, 269-274.

(14) Manaa, M. R.; Yarkony, D. R. On the intersection of two potential energy surfaces of the same symmetry. Systematic characterization using a Lagrange multiplier constrained procedure. J. Chem. Phys. 1993, 99, 5251-5256. 
(15) Li, Q.; Mendive-Tapia, D.; Paterson, M. J.; Migani, A.; Bearpark, M. J.; Robb, M. A.; Blancafort, L. A global picture of the $\mathrm{S}_{1} / \mathrm{S}_{0}$ conical intersection seam of benzene. Chem. Phys. 2010, 377, 60-65.

(16) Mori, T.; Martínez, T. J. Exploring the Conical Intersection Seam: The Seam Space Nudged Elastic Band Method. J. Chem. Theory Comput. 2013, 9, 1155-1163.

(17) Maeda, S.; Ohno, K.; Morokuma, K. Automated Global Mapping of Minimal Energy Points on Seams of Crossing by the Anharmonic Downward Distortion Following Method: A Case Study of $\mathrm{H}_{2}$ CO. J. Phys. Chem. A 2009, 113, 1704-1710.

(18) Maeda, S.; Saito, R.; Morokuma, K. Finding Minimum Structures on the Seam of Crossing in Reactions of Type $\mathrm{A}+\mathrm{B} \rightarrow \mathrm{X}$ : Exploration of Nonadiabatic Ignition Pathways of Unsaturated Hydrocarbons. J. Phys. Chem. Lett. 2011, 2, 852-857.

(19) Harabuchi, Y.; Maeda, S.; Taketsugu, T.; Minezawa, N.; Morokuma, K. Automated Search for Minimum Energy Conical Intersection Geometries between the Lowest Two Singlet States $\mathrm{S}_{0} / \mathrm{S}_{1}$-MECIs by the Spin-Flip TDDFT Method. J. Chem. Theory Comput. 2013, 9, 4116-4123.

(20) Maeda, S.; Taketsugu, T.; Ohno, K.; Morokuma, K. From Roaming Atoms to Hopping Surfaces: Mapping Out Global Reaction Routes in Photochemistry. J. Am. Chem. Soc. 2015, 137, 3433-3445.

(21) Sicilia, F.; Bearpark, M. J.; Blancafort, L.; Robb, M. A. An analytical second-order description of the $\mathrm{S}_{0} / \mathrm{S}_{1}$ intersection seam: fulvene revisited. Theor. Chem. Acc. 2007, $118,241-251$.

(22) Sicilia, F.; Blancafort, L.; Bearpark, M. J.; Robb, M. A. New Algorithms for Optimizing and Linking Conical Intersection Points. J. Chem. Theory Comput. 2008, 4, 257-266. 
(23) Laio, A.; Parrinello, M. Escaping free-energy minima. Proc. Nat. Acad. Sci. USA 2002, 99, 12562-12566.

(24) Barducci, A.; Bussi, G.; Parrinello, M. Well-tempered metadynamics: a smoothly converging and tunable free-energy method. Phys. Rev. Lett. 2008, 100, 020603.

(25) Raiteri, P.; Laio, A.; Gervasio, F. L.; Micheletti, C.; Parrinello, M. Efficient Reconstruction of Complex Free Energy Landscapes by Multiple Walkers Metadynamics. J. Phys. Chem. B 2006, 110, 3533-3539.

(26) Bonomi, M.; Parrinello, M. Enhanced Sampling in the Well-Tempered Ensemble. Phys. Rev. Lett. 2010, 104.

(27) Tribello, G. A.; Ceriotti, M.; Parrinello, M. A self-learning algorithm for biased molecular dynamics. Proc. Nat. Acad. Sci. USA 2010, 107, 17509-17514.

(28) Branduardi, D.; Bussi, G.; Parrinello, M. Metadynamics with Adaptive Gaussians. J. Chem. Theory Comput. 2012, 8, 2247-2254.

(29) Lindner, J. O.; Röhr, M. I. S.; Mitrić, R. Multistate metadynamics for automatic exploration of conical intersections. Phys. Rev. A 2018, 97, 052502.

(30) Ceriotti, M.; Tribello, G. A.; Parrinello, M. Simplifying the representation of complex free-energy landscapes using sketch-map. Proc. Nat. Acad. Sci. USA 2011, 108, 1302313028.

(31) Olivucci, M.; Ragazos, I. N.; Bernardi, F.; Robb, M. A. A conical intersection mechanism for the photochemistry of butadiene. A MC-SCF study. J. Am. Chem. Soc. 1993, $115,3710-3721$.

(32) Bernardi, F.; Olivucci, M.; Robb, M. A. The role of conical intersections and excited state reaction paths in photochemical pericyclic reactions. J. Photoch. Photobio. A 1997, 105, 365-371. 
(33) Krawczyk, R. P.; Malsch, K.; Hohlneicher, G.; Gillen, R. C.; Domcke, W. $1^{1} \mathrm{~B}_{u}-2^{1} \mathrm{~A}_{g}$ conical intersection in trans-butadiene: ultrafast dynamics and optical spectra. Chem. Phys. Lett. 2000, 320, 535-541.

(34) Maeda, S.; Harabuchi, Y.; Taketsugu, T.; Morokuma, K. Systematic Exploration of Minimum Energy Conical Intersection Structures near the Franck-Condon Region. $J$. Phys. Chem. A 2014, 118, 12050-12058.

(35) Palmer, I. J.; Ragazos, I. N.; Bernardi, F.; Olivucci, M.; Robb, M. A. An MC-SCF study of the $\mathrm{S}_{1}$ and $\mathrm{S}_{2}$ photochemical reactions of benzene. J. Am. Chem. Soc. 1993, $115,673-682$.

(36) Lasorne, B.; Bearpark, M. J.; Robb, M. A.; Worth, G. A. Controlling $\mathrm{S}_{1} / \mathrm{S}_{0}$ Decay and the Balance between Photochemistry and Photostability in Benzene: A Direct Quantum Dynamics Study. J. Phys. Chem. A 2008, 112, 13017-13027.

(37) Parker, D.; Minns, R.; Penfold, T.; Worth, G.; Fielding, H. Ultrafast dynamics of the $\mathrm{S}_{1}$ excited state of benzene. Chem. Phys. Lett. 2009, 469, 43-47.

(38) Mihalić, Z.; Nikolić, S.; Trinajstić, N. Comparative study of molecular descriptors derived from the distance matrix. J. Chem. Inf. Model. 1992, 32, 28-37.

(39) Bogdanov, B.; Nikolić, S.; Trinajstić, N. On the three-dimensional wiener number. J. Math. Chem. 1989, 3, 299-309.

(40) Cremer, D.; Pople, J. A. General definition of ring puckering coordinates. J. Am. Chem. Soc. 1975, 97, 1354-1358.

(41) Werner, H.-J.; Knowles, P. J.; Knizia, G.; Manby, F. R.; Schütz, M. Molpro: a generalpurpose quantum chemistry program package. WIREs Comput. Mol. Sci. 2011, 2, 242-253. 
(42) Ditchfield, R.; Hehre, W. J.; Pople, J. A. Self-Consistent Molecular-Orbital Methods. IX. An Extended Gaussian-Type Basis for Molecular-Orbital Studies of Organic Molecules. J. Chem. Phys. 1971, 54, 724-728.

(43) Hariharan, P. C.; Pople, J. A. The influence of polarization functions on molecular orbital hydrogenation energies. Theor. Chim. Acta 1973, 28, 213-222.

(44) Koslowski, A.; Beck, M. E.; Thiel, W. Implementation of a general multireference configuration interaction procedure with analytic gradients in a semiempirical context using the graphical unitary group approach. J. Comput. Chem. 2003, 24, 714-726.

(45) Patchkovskii, S.; Koslowski, A.; Thiel, W. Generic implementation of semi-analytical CI gradients for NDDO-type methods. Theor. Chem. Acc. 2005, 114, 84-89.

(46) Petersen, J.; Wohlgemuth, M.; Sellner, B.; Bonačić-Kouteckỳ, V.; Lischka, H.; Mitrić, R. Laser pulse trains for controlling excited state dynamics of adenine in water. Phys. Chem. Chem. Phys. 2012, 14, 4687-4694.

(47) Verlet, L. Computer "Experiments" on Classical Fluids. I. Thermodynamical Properties of Lennard-Jones Molecules. Phys. Rev. 1967, 159, 98-103.

(48) Berendsen, H. J. C.; Postma, J. P. M.; van Gunsteren, W. F.; DiNola, A.; Haak, J. R. Molecular dynamics with coupling to an external bath. J. Chem. Phys. 1984, 81, 36843690.

(49) Bussi, G.; Branduardi, D. Free-Energy Calculations with Metadynamics: Theory and Practice. Rev. Comput. Chem. 2015, 28, 1-49.

(50) Barbatti, M.; Lischka, H. Nonadiabatic Deactivation of $9 H$-Adenine: A Comprehensive Picture Based on Mixed Quantum-Classical Dynamics. J. Am. Chem. Soc. 2008, 130, 6831-6839. 
(51) Perun, S.; Sobolewski, A. L.; Domcke, W. Ab Initio Studies on the Radiationless Decay Mechanisms of the Lowest Excited Singlet States of 9H-Adenine. J. Am. Chem. Soc. 2005, 127, 6257-6265. 


\section{Graphical TOC Entry}

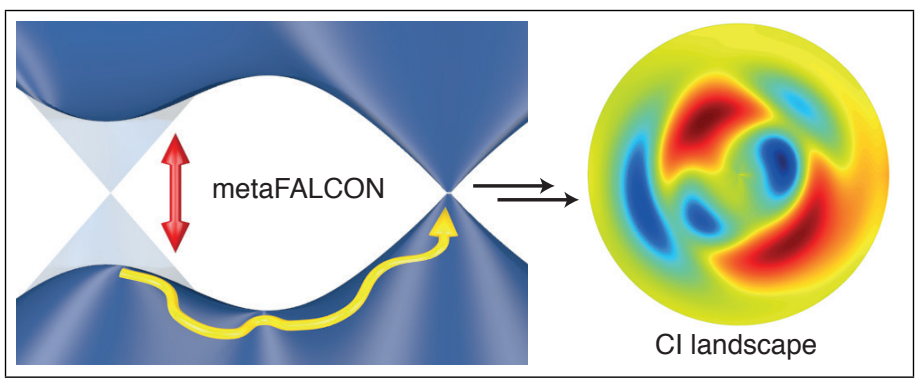

